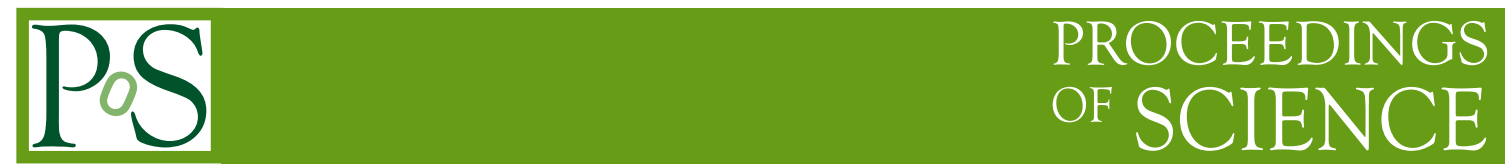

\title{
Hadronic Decays
}

\section{Michael*}

Theoretical Physics Division, Dept of Mathematical Sciences, University of Liverpool, Liverpool L69 3BX, UK

E-mail: c.michaeleliv.ac.uk

Hadronic decays and transitions are a key ingredient of hadronic physics. I discuss how hadronic decays can be explored in lattice gauge theory and review studies undertaken. I also discuss the impact of decays on masses and how lattice studies can explore the nature of a hadronic state: namely whether it is a molecular or quark-antiquark state. A brief discussion of lattice exploration of pentaquark states is presented.

XXIII International Symposium on Lattice Field Theory

25th-30th July 2005

School of Mathematics, Trinity College, Dublin

* Speaker. 


\section{Introduction}

Relatively few hadronic states are stable to strong decays (i.e. via QCD with degenerate $u$ and $d$ quarks). Among the mesons, we have [1]:

$\begin{array}{ll}\text { Stable } & \pi K \eta D D_{s} B B_{s} B_{c} D_{s}^{*} B^{*} B_{s}^{*} D_{s}\left(0^{+}\right) B_{s}\left(0^{+}\right) \\ \Gamma<1 \mathrm{MeV} & \eta^{\prime} D^{*} \psi(1 S) \psi(2 S) \chi_{1} \chi_{2} \Upsilon(1 S) \Upsilon(2 S) \Upsilon(3 S) \\ \Gamma<10 \mathrm{MeV} & \omega \phi \chi_{0} X(3872) \\ \Gamma>10 \mathrm{MeV} & \rho f_{0} a_{0} h_{1} b_{1} a_{1} f_{2} f_{1} a_{2}, \text { etc., inc } \eta_{c} .\end{array}$

The mass of an unstable state is usually defined as the energy corresponding to a $90^{0}$ phase shift. This definition seems to accord with simple mass formulae: For example

- $\rho(776)$ and $\omega(783)$ are close in mass despite having widths of 150 and $8 \mathrm{MeV}$ respectively.

- The baryon decuplet $(\Delta(1232), \Sigma(1385), \Xi(1530), \Omega(1672))$ is roughly equally spaced in mass despite having widths of $(120,37,9,0) \mathrm{MeV}$ respectively.

(Note that defining the mass as the real part of the pole will cause a downward shift of masses for wider states, eg. $22 \mathrm{MeV}$ less [2] for the $\Delta(1232)$ pole, and this prescription will fit the equal mass rule less well.)

So, on the one hand, unstable particles seem to fit in well with stable ones; on the other hand, the presence of open decay channels will have an influence in lattice studies.

Some of the motivations to study hadronic decays on the lattice are:

- To determine properties of exotic states (glueball; hybrid meson; multi-quark) to guide experiment.

- To understand the nature of states: whether meson-meson or quark-antiquark in structure.

- To understand the impact of decay on the mass of a state.

- Hadronic decays are now accessible in full QCD: for example $\rho_{0} \rightarrow \pi_{1} \pi_{-1}$ at rest requires

$$
\frac{m(\pi)}{m(\rho)}<\frac{0.5}{\sqrt{1+\frac{4 \pi^{2}}{(m(\pi) L)^{2}}}}
$$

so for $m(\pi) L=5$ this implies $m(\pi) / m(\rho)<0.32$, but note that $\rho_{1} \rightarrow \pi_{1} \pi_{0}$, where suffix labels momentum in units of $2 \pi / L$, only needs $m(\pi) / m(\rho)=0.44$,

\section{Decays in Euclidean Time}

NO GO. At large spatial volume, the two-body continuum masks any resonance state. The extraction of the spectral function from the correlator $C(t)$ is ill-posed unless a model is made [3, 4], since the low energy continuum dominates at large $t$. 
GO. For finite spatial volume $\left(L^{3}\right)$, the two-body continuum is discrete and Lüscher showed [5, $6,7]$ how to use the small energy shifts with $L$ of these two-body levels to extract the elastic scattering phase shifts. The phase shifts then determine the resonance mass and width, see ref. [8] for a review. Thus a relatively broad resonance such as the $\rho$ appears as a distortion of the $\pi_{n} \pi_{-n}$ energy levels where pion momentum $q=2 \pi n / L$.

The effect can be visualised as arising from the relatively larger amplitude for interaction between two hadrons in increasingly smaller spatial volumes:
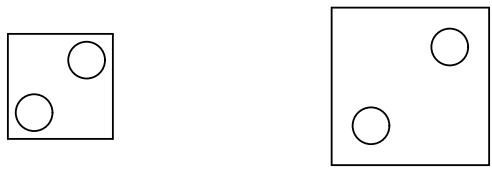

\section{Lattice evaluation}

Lüscher's method is applicable in principle to study phase shifts below inelastic thresholds. But problems arise in practical implementation:

- Accurate measurement of a small energy difference (of order $L^{-3}$ in general, though bigger at resonance) is needed.

- Measurement of a matrix of correlations between two-body and one-body operators will be needed to get accurate energy values and these correlators are less straightforward to evaluate.

- Measurements are needed at several spatial sizes $(L)$ with dynamical fermions, with $L$ big enough to hold two hadrons.

- Higher energy levels in a given channel are particularly hard to determine (eg. $\pi_{1} \pi_{-1}$ compared to $\pi_{0} \pi_{0}$ ) since excited state energies are always difficult to extract with precision from temporal correlators, since the ground state contribution dominates at larger $t$.

Are there any short cuts?

\section{Lattice evaluation: tricks}

\section{$4.1 \pi^{+} \pi^{+}$}

One of the first cases that has been explored is the $\pi^{+} \pi^{+}$interaction at low energy. This is favourable since there are fewer quark diagrams to evaluate as there is no annihilation diagram, i.e no $\bar{q} q$ channel and, because of this, quenched evaluation is a useful approximation. This has been much studied in the last decades. Indeed recently there have also been dynamical quark evaluations $[9,10]$ of the $\pi^{+} \pi^{+}$scattering near threshold.

The trick that has been evolved to improve precision in these studies is that of using an operator that creates two pions at spatial separation $x$. Then the nature of the expected spatial wave function 
versus $x$ allows to determine the scattering length (phase shift $\delta_{I=2}$ near threshold) more accurately than from the energy determined by the $t$-correlation alone [11].

This $\pi^{+} \pi^{+}$case does not involve decay, of course, just an evaluation of the hadronic interaction strength. To explore decays, one must study the transition between two meson and one meson operators.

\subsection{Hadronic transitions}

Consider a lattice study of the off-diagonal correlator: from a $\rho$ meson to $\pi \pi$. Diagrammatically:
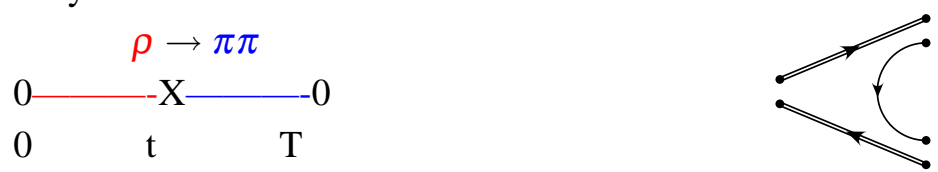

Now to evaluate this contribution, since the intermediate point marked $\mathrm{X}$ at time $t$ is not observed on a lattice, it must be summed over.

$$
\sum_{t=0}^{T} e^{-m(\rho) t} x e^{-m(\pi \pi)(T-t)} \rightarrow A e^{-m(\rho) T}-B e^{-m(\pi \pi) T}
$$

The problem that arises is that excited states of either the $\rho$ or of $\pi \pi$ will contribute a similar behaviour:

$$
\begin{aligned}
& \sum_{t=0}^{T} e^{-m^{\prime}(\rho) t} x e^{-m(\pi \pi)(T-t)} \rightarrow C e^{-m^{\prime}(\rho) T}-D e^{-m(\pi \pi) T} \\
& \sum_{t=0}^{T} e^{-m(\rho) t} x e^{-m^{\prime}(\pi \pi)(T-t)} \rightarrow E e^{-m(\rho) T}-F e^{-m^{\prime}(\pi \pi) T}
\end{aligned}
$$

Hence there is no way in principle to remove any excited state contamination unless $m(\rho) \approx$ $m(\pi \pi)$ when the ground state piece sums to $x T e^{-m T}$ while excited states only behave as $e^{-m T}$.

Thus for on-shell transitions on the lattice, it is possible to extract the hadronic transition amplitude directly $[12,13,14]$. The key signal is to observe a linear dependence of the lattice normalised transition amplitude on the temporal extent $T$. The slope of this transition then gives the lattice amplitude $x$ that can be related to the transition amplitude with conventional normalisation of states.

\section{Lattice evaluation: $\rho \rightarrow \pi \pi$}

This transition can be evaluated directly - for on shell transitions - by looking for the signal extensive in $T$. This has been explored [14] for $\rho$ decay to $\pi \pi$. In order to have an on-shell transition with the dynamical quark lattices then available, it was optimum to study the case of decay in flight: $\rho_{1} \rightarrow \pi_{1} \pi_{0}$ which is quite close to on-shell as illustrated in fig. 1 . The diagrams illustrated in fig. 1 were evaluated using a stochastic time-plane source method. The generalisation of Lüscher's method to decay in flight is given in [15]. 

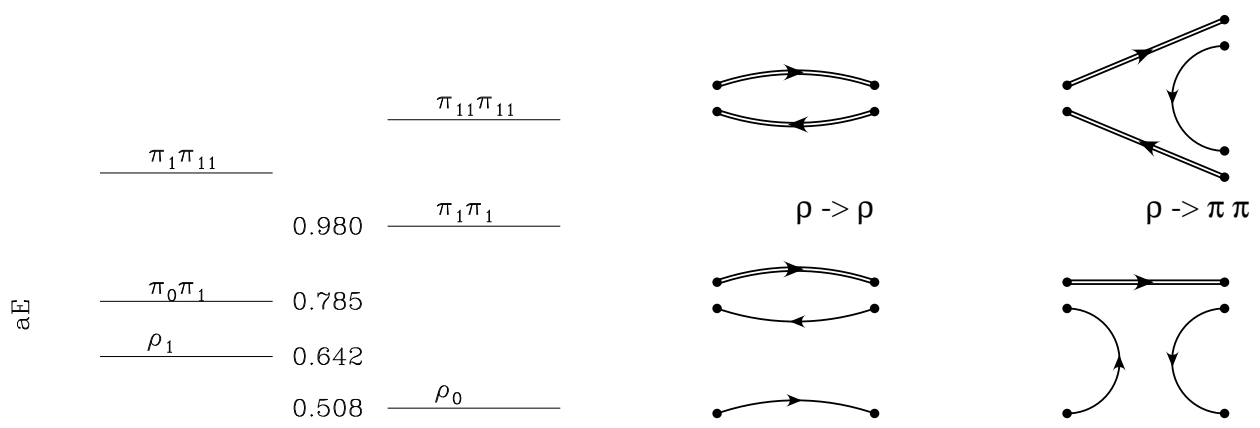

$\rho-\rho$

$\rho \rightarrow \pi \pi$
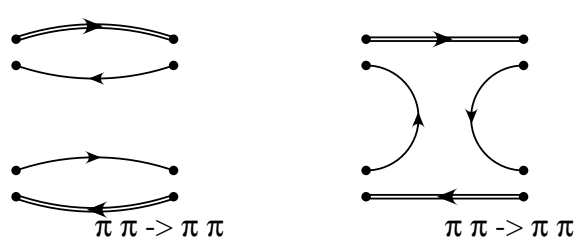

Figure 1: (a) Energy levels of $\rho$ meson and $\pi \pi$ states. From UKQCD with $N_{f}=2$ sea quarks with NPimproved fermions, $m(\pi) / m(\rho)=0.58, m(\pi) L=4.6, a=0.11 \mathrm{fm}$. (b) Diagrams evaluated (stochastic timeplane source method)
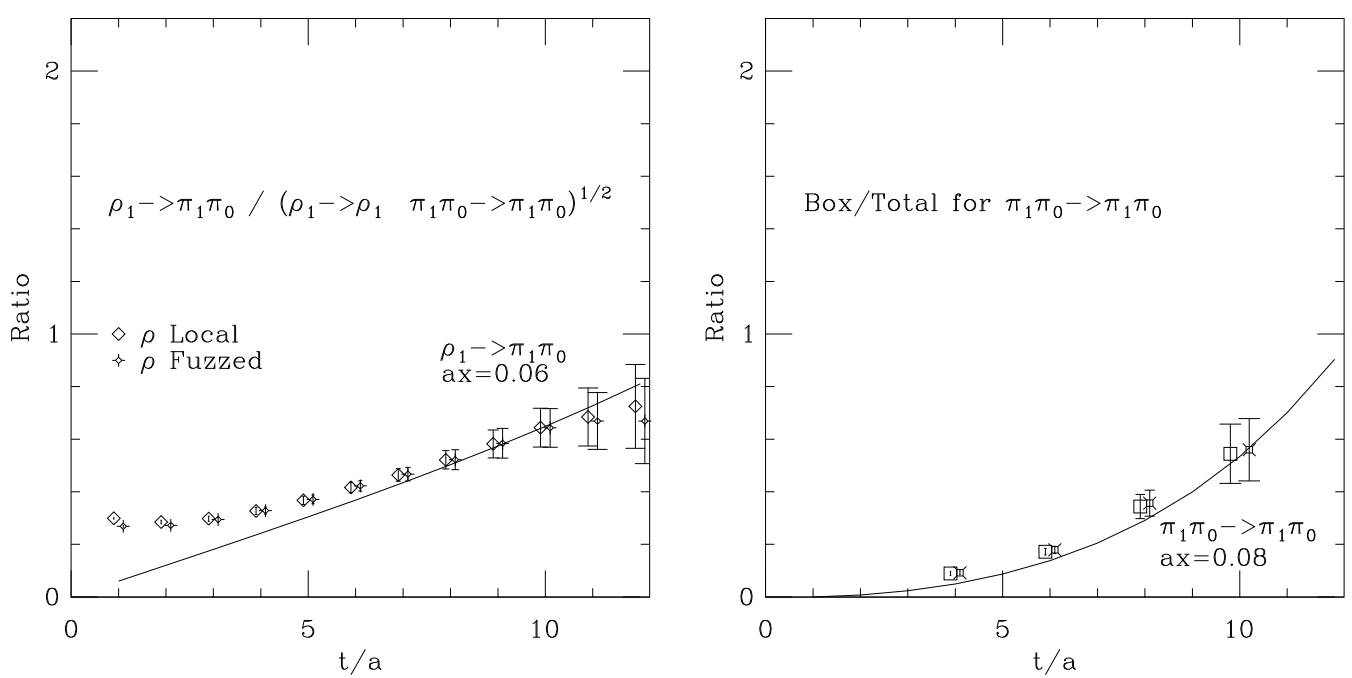

Figure 2: Strength of transitions measured on the lattice.

The signal obtained from the lattice study is illustrated in fig. 2 both for the normalised $\rho \rightarrow$ $\pi \pi$ transition and for the normalised "box" contribution $\pi \pi \rightarrow \pi \pi$ which has a contribution from $\pi \pi \rightarrow \rho \rightarrow \pi \pi$. The approximate consistency of these two approaches is a useful cross-check.

Assuming two close energy levels (with mixing amplitude $x$ ) mix to give an energy shift $\Delta m$, then $m\left(\rho_{1}\right)$ moves down, while $E\left(\pi_{1} \pi_{0}\right)$ moves up. The lattice results are

- Transition $\rho_{1} \rightarrow \pi_{1} \pi_{0}$ : Signal $x T$. $a \Delta m=0.022_{-7}^{+17}$

- Box $\pi_{1} \pi_{0} \rightarrow \pi_{1} \pi_{0}$ : Signal $(x T)^{2}$. (consistent with above)

- Shift of energy of $\rho_{1}$ spin parallel to momentum (which mixes with $\pi_{1} \pi_{0}$ ) to spin perpendicular to momentum (which does not). $a \Delta m=0.026(7)$ 
- Lüscher shift (unbinding energy of $\pi_{1} \pi_{0}$ ). $a \Delta m=0.04(3)$. Note big error

So the tricks give smaller errors than the direct determination of the Lüscher mass shift. The tricks have some inherent systematic errors, however, and are most useful for a qualitative study.

These mass shifts with the assumption of $\rho$ dominance of the $\pi \pi$ partial wave give a determination of the coupling $g_{\rho \pi \pi}$. Thus one can determine this coupling constant from the lattice (where decay does not proceed) and compare with experiment:

\begin{tabular}{llll}
\hline method & $m_{\text {val }}$ & $m_{\text {sea }}$ & $\bar{g}$ \\
\hline Lattice $x T$ & $s$ & $s$ & $1.40_{-23}^{+47}$ \\
Lattice $\rho$ shift & $s$ & $s$ & $1.56_{-13}^{+21}$ \\
\hline$\phi \rightarrow K \bar{K}$ & $s$ & $u, d$ & 1.5 \\
$K^{*} \rightarrow K \pi$ & $u, d / s$ & $u, d$ & 1.44 \\
$\rho \rightarrow \pi \pi$ & $u, d$ & $u, d$ & 1.39 \\
\hline
\end{tabular}

The agreement is good, indicating that there is a relatively mild dependence of the coupling constant on the sea quark mass (which is higher in the lattice study than in experiment). This satisfactory confrontation between lattice and experiment, is encouraging for lattice exploration of cases where predictions need to be made since experimental data are not available.

\section{Hybrid meson decay}

One of the characteristic predictions of QCD is that there can be mesons in which the gluonic degrees of freedom are non-trivially excited. The simplest example is a hybrid meson with spinexotic $J^{P C}=1^{-+}$which is a $J^{P C}$ combination not available to a $\bar{q} q$ state.

\subsection{Heavy quarks}

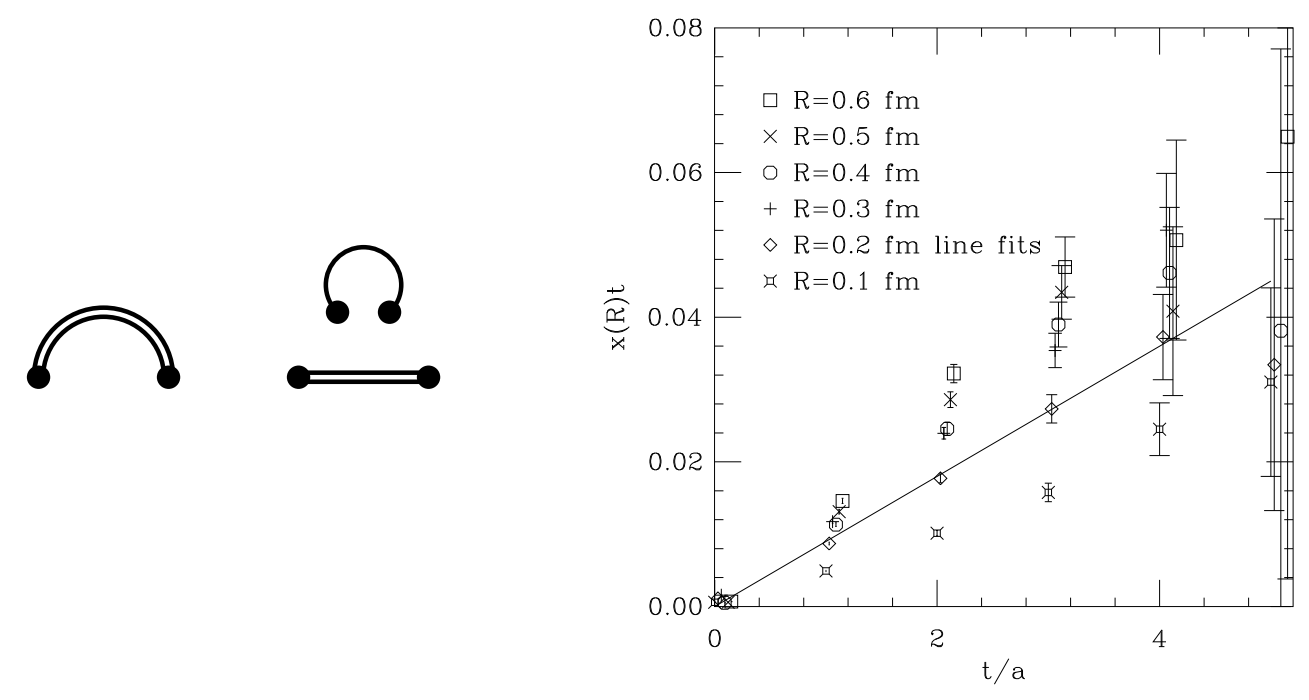

Figure 3: Strength of string de-excitation transitions measured on the lattice. 
The cleanest environment in which to study such states on a lattice is in the limit of very heavy quarks $-\bar{b} b$. This can be approximated by using static quarks and the gluonic excitation arises as an excited string state between these static quarks with non-trivial gluonic angular momentum. Lattice studies have long predicted the spectrum of such states.

To guide experiment, however, it is important to know the expected decay mechanism and associated width. In the static quark limit, several symmetries can be used which imply [16] that the dominant decay will be string de-excitation (rather than string breaking). Lattice study [16] shows that the dominant decay of $H_{b}$ is string de-excitation to $\chi_{b} f_{0}$. The transition that is considered is shown by the diagram on the left of fig. 3 . The lattice data on the transition are illustrated in fig. 3 : the transition is closest to on-shell for $R \approx 0.2 \mathrm{fm}$. The width is predicted to be around $80 \mathrm{MeV}$.

This estimate from first principles of the decay width is of significance in guiding experimental searches for such hybrid states.

\subsection{Light quarks}

For light quarks, evidence for contributions from two-body states (such as $\pi b_{1}$ ) to the spinexotic $\left(J^{P C}=1^{-+}\right)$channel is seen in dynamical studies [17] - this complicates the extraction of a spin-exotic hybrid meson.

A recent exploratory study (quenched) of $\pi a_{1}$ mixing with the $J^{P C}=1^{-+}$spin-exotic hybrid meson has given an estimate of the width for this decay [18].

\section{String breaking}

In quenched QCD, as the static quark and antiquark sources are pulled apart the potential energy rises as $\sigma R$, where $\sigma$ is the string tension. In full QCD, a sea quark-antiquark can be created from the vacuum and it becomes energetically favourable to have a meson-antimeson pair beyond some value of $R$. This is the phenomenon of string breaking. It has long been realised that this can be studied as a mixing phenomenon [19] with channels $Q \bar{Q}$ and $Q \bar{q} q \bar{Q}$. For static quarks at separation $R$, there will be a level crossing and associated mixing of $V(R)$ and $2 m(B)$. This mixing is the measure of string breaking [13], [20]. This mixing energy is very hard to determine on a lattice: since the crossing occurs at relatively large $R \approx 1.25 \mathrm{fm}$ where amplitudes are small. At this crossing point, for static quarks, there is a mixing transition which will be independent of lattice spatial size $L$ for sufficiently large $L$ since each state is localised (the heavy quarks are static).

Employing "all the tricks in the book" a first estimate of this energy shift (mixing amplitude) has been obtained [21] of 51(3) MeV. An illustration of the crossing region is in fig. 4.

Using the adiabatic approximation, this energy gap can be used to evaluate amplitudes for excited $\Upsilon$ decay to $B \bar{B}$. So it does indeed have some relevance to the topic of hadronic decays.

\section{Scalar Mesons}

\subsection{Light quarks}

Since $u \bar{u}+d \bar{d}, s \bar{s}$, glueball, and meson-meson components are all possible for flavour-singlet scalar mesons, this is a difficult area to study both on a lattice, and in interpreting experimental 


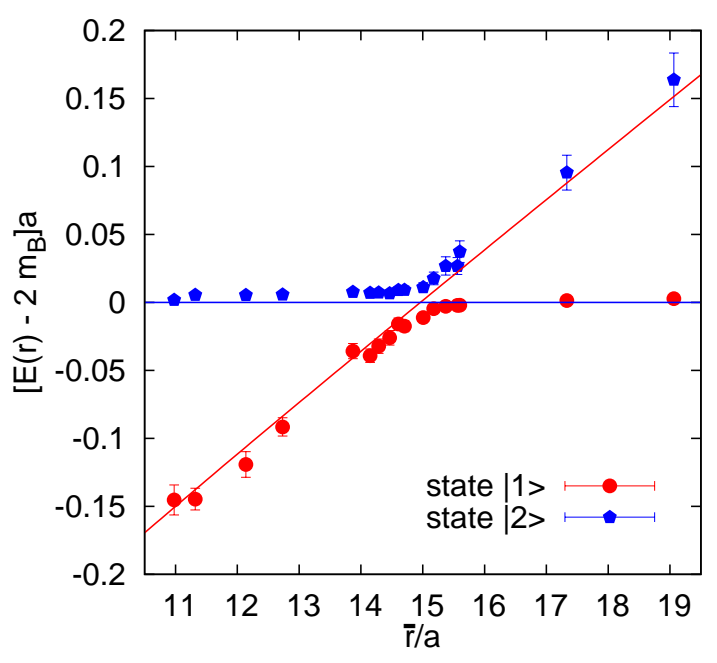

Figure 4: String breaking (here $a \approx 0.083 \mathrm{fm}$ ).

data. For scalar mesons the lowest mass decay channels are $\pi \pi$ (flavour singlet: $f_{0}$ ) or $\eta \pi$ (flavour non-singlet: $a_{0}$ ) and these decay channels are open in many dynamical lattice studies. The history of attempts to study the complex mixing between these different contributions is :

- $0^{++}$Glueball decay $\rightarrow \pi \pi$ : quenched study [22, 23].

- Glueball mixing with $q \bar{q}$ meson. This is a hadronic transition which is independent of $L$ for large $L$, so a mixing energy can be quoted. It has been studied using quenched [24] and dynamical lattices [12]

A full lattice study is needed which includes glueball, $\bar{q} q$ and $\pi \pi$ channels but the disconnected diagram for $f_{0} \rightarrow \pi \pi$ is very noisy in practice. To reduce the contribution from disconnected diagrams, one can study flavour non-singlet decays. The simplest case is $a_{0} \rightarrow \eta \pi$ and this has been explored in quenched studies which have an anomalous behaviour: since the $\eta$ itself is unphysical (appearing as a double pole degenerate in mass with the pion). Rather than try to correct for this anomaly which gives a wrong sign to the $a_{0}$ correlator at larger $t$, it is preferable to use a ghost-free theory. With two flavours of sea quark $\left(N_{f}=2\right)$, the $a_{0} \rightarrow \eta \pi$ transition is physical but it involves the evaluation of an additional disconnected diagram - as illustrated in fig. 5. This transition is then approximately on-shell and the transition strength can be evaluated, as shown in fig. 5 .

The results [25] are indeed as anticipated for the transition involving particles with no momentum - showing an approximately linear rise. For the decay in flight the result is significantly different even though, for an S-wave transition, one would expect a transition amplitude independent of momentum. This is perhaps a warning that the underlying dynamics is more complicated. Further study is needed in this area of light-light scalar mesons.

\subsection{Heavy-light quarks}

One of the most promising areas to study scalar mesons on lattice is for heavy-light mesons. The scalar meson with $\bar{c} s$ quantum number is known experimentally [1] to be very narrow (it decays 

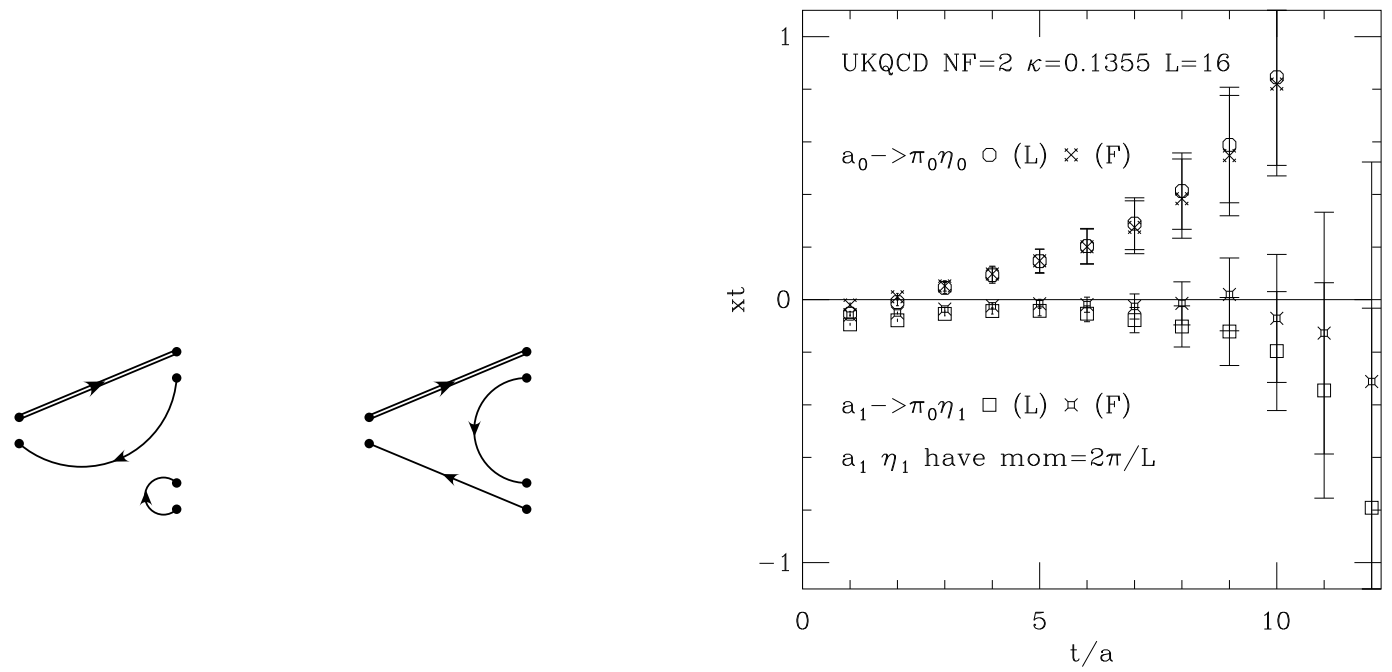

Figure 5: Diagrams for $a_{0} \rightarrow \eta \pi$ and transition strength on a lattice (preliminary results from McNeile et al. [25]).

only via the isospin-violating channel $D_{s} \pi$ or electromagnetically), while the scalar meson with $\bar{b} s$ quantum number is predicted to be similarly narrow from a lattice study of its energy [26].

The heavy-light scalar meson, which in the limit of a static heavy quark, is expected to be stable [26] for $\bar{b} s$ content and to decay to $B \pi$ for $\bar{b} n$ content (where $n=u, d$, considered as degenerate). Evaluating the diagram shown in fig. 6, a lattice estimate of the decay rate of $B\left(0^{+}\right) \rightarrow B\left(0^{-}\right) \pi$ gives a width predicted [27] as $162(30) \mathrm{MeV}$. This state has not been observed experimentally yet, but the experimental results for the corresponding $\bar{c} n$ state, $D\left(0^{+}\right)$, are that the width is $270 \pm 50 \mathrm{MeV}$. Although significant $1 / m_{Q}$ effects are expected in the HQET in extrapolating to charm quarks, this is indeed a similar magnitude to that predicted for $B$ mesons. It will be interesting so see how the lattice prediction of the mass and width of the $B\left(0^{+}\right)$fares when experimental results are available.
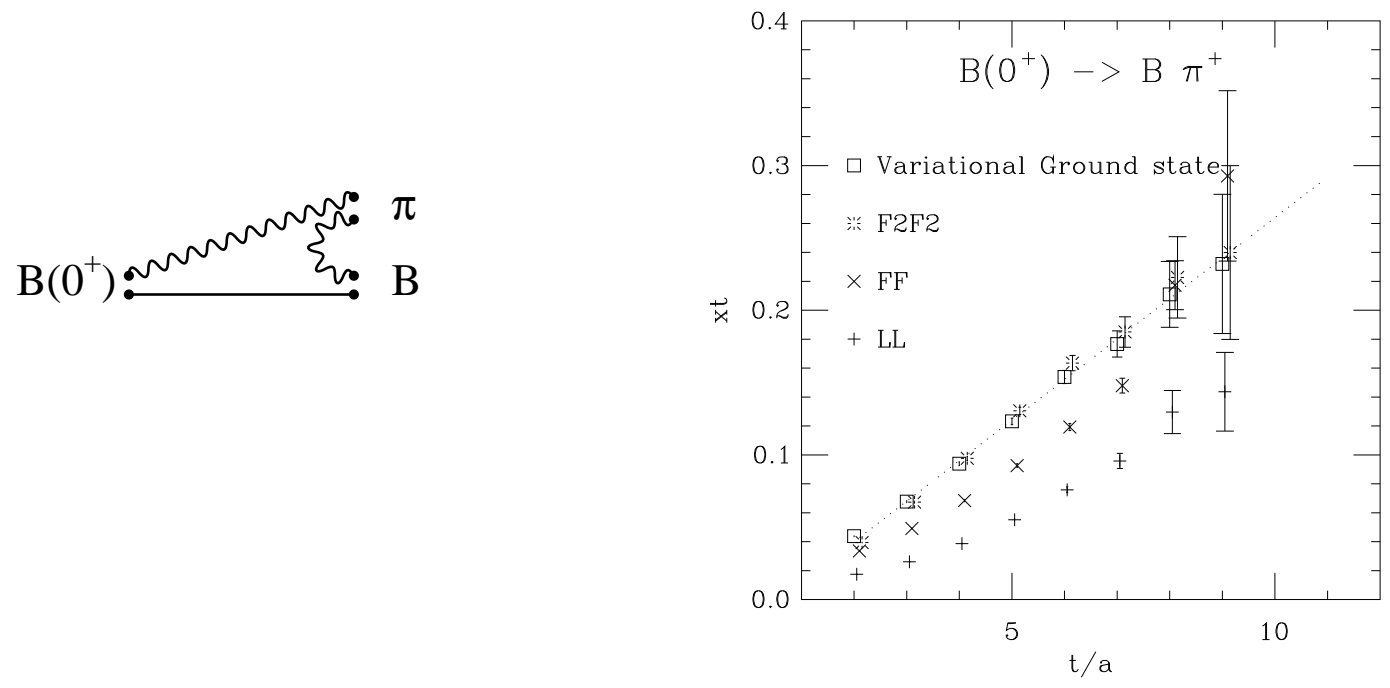

Figure 6: Diagrams for $B\left(0^{+}\right) \rightarrow B \pi$ and transition strength on a lattice. 


\section{Do decays matter?}

The previous discussion of decays on a lattice emphasises that $q \bar{q}$ states do mix with two-body states with the same quantum numbers. In the real world, the two-body states are a continuum and nearby states have a predominant influence. Since there is a suppression in the amplitude near threshold from the factor $q^{L}$ for an $L$-wave transition, for S-wave transitions $(L=0)$ the threshold will turn on most abruptly and hence will have stronger mixing.

For bound states there is an influence of nearby many-body states (eg. $N \pi$ on $N$ or $\pi \pi \pi$ on $\pi$ ) which mix to reduce the mass. The nearest such thresholds will be those with pionic channels since pions are the lightest mesons. This is the province of low energy effective theories, especially Chiral Perturbation Theory which is discussed in other talks. This then provides a reliable guide in extrapolating lattice results to the physical light quark masses.

For unstable states (resonances) the influence of the two-body continuum is less clear since the two-body states are both lighter and heavier. In the continuum at large volume, effective field theories can again be used to explore this. On a lattice, however, the signal for a particle becomes obscured as the quark mass is reduced so that it becomes unstable. Techniques, such as those discussed above, are needed to extract the elastic scattering phase shift and hence the mass and width.

For quenched QCD, however, where these two-body states are not coupled (or have the wrong sign as in $a_{0} \rightarrow \eta \pi$ ), then the unstable states will be distorted compared to full QCD. For instance, in existing quenched QCD studies, the $\rho$ will be too heavy since it is not repelled by the heavier $\pi \pi$ states. Indeed an example of this effect was seen above in the study of $\rho$ decay including dynamical sea quarks, where the $\rho$ mass decreased [14] when it could couple to $\pi \pi$ compared to when it could not.

\section{Molecular states?}

Can lattice QCD provide evidence about possible molecular states: hadrons made predominantly of two hadrons?

The prototype is the deuteron: $n p$ bound in a relative S-wave (with some $\mathrm{D}$-wave admixture) by $\pi$ exchange.

There are many states close to two-body thresholds. Since S-wave thresholds are the most abrupt, it is usually in this case that the influence of the threshold on the state has been discussed. Some of these cases are:

$$
\begin{aligned}
& f_{0}(980) a_{0}(980) \leftrightarrow K \bar{K} \quad D_{s}\left(0^{+}\right) \leftrightarrow \quad D\left(0^{-}\right) K \\
& B_{s}\left(0^{+}\right) \leftrightarrow B\left(0^{-}\right) K \quad X(3872) \quad \leftrightarrow \quad D^{*} \bar{D} \\
& \Lambda(1405) \leftrightarrow \bar{K} N \quad N(1535) \leftrightarrow \eta N
\end{aligned}
$$

Some of these states $\left(D_{s}\left(0^{+}\right), B_{s}\left(0^{+}\right)\right)$are stable (in QCD in the isospin conserving limit) whereas the rest have other channels open. There is a large literature, stretching over 40 years, discussing the consequences of the nearby threshold on these states. One definite implication is that isospin breaking is enhanced by mass splittings in thresholds (eg. $\overline{K^{0}} K^{0}$ compared to $K^{+} K^{-}$is 
$8 \mathrm{MeV}$ higher and this induces isospin mixing between the states at $980 \mathrm{MeV}$ ). This level of detail is not accessible in lattice studies at present, but lattice QCD should be able to address the issue of the influence of thresholds on these states.

The observation of a state near a 2-body threshold implies that there is an attractive interaction between the two bodies. But this is a topic like that of whether the chicken or egg was created first: an attractive interaction implies and is implied by a nearby state. What can lattice QCD offer here? We are in the position of being able to vary the quark masses and this is a very useful tool. A two-body threshold will move in general in a different way with changing quark mass than a $\bar{q} q$ state. We can also move the strange and non-strange masses separately and this can be helpful too.

Another line of investigation is that lattice studies can explore the wavefunction of a state either the Bethe-Salpeter wavefunction or the charge or matter spatial distribution. One can also explore the coupling of a state to a 2-body channel, as was discussed above.

The prototype of a molecular state is the deuteron: it has a tiny binding energy $(2.2 \mathrm{MeV})$ and a very extended spatial wave function. Pion exchange between neutron and proton gives a mechanism for this long-range attraction. In general it is difficult to reproduce such small binding energies in lattice studies.

Another case where a long-range pion exchange can give binding is in the $B B$ system. Here lattice results indicate [28] the possibility of molecular bound states in some quantum number channels which have an attractive interaction from pion exchange, but also the possibility of bound multi-quark states which are not described as hadron-hadron but where the two heavy quarks form a colour triplet and the light quarks are arranged as in a heavy-light-light baryon. This $B B$ example illustrates the rich structure available to multi-quark systems.

Cases that have been studied on the lattice are the $\bar{b} s$ and $\bar{c} s$ scalar mesons [26, 29]. The lattice mass values do suggest that these states, treated as $\bar{Q} q$ states, are bound and are not unduly influenced by the $B K, D K$ threshold. A study of the charge distribution of the $B\left(0^{+}\right)$gives $[30,31]$ confirmation since the light quark spatial distribution is similar to that of other $\bar{Q} q$ states.

For the $a_{0}$ and $f_{0}$ at $980 \mathrm{MeV}$, there is limited progress. Quenched studies are inappropriate here for the $a_{0}$ since the $\eta \pi$ decay is wrongly treated. In dynamical studies [12,32], the two body channels are of similar energy to the $a_{0}$ (as discussed above), so analysis is unclear. A thorough study of the light-light scalar sector is still awaited.

\section{Multi-quark states?}

I told you so: a narrow pentaquark above $K N$ threshold is not possible in QCD. I lived through the split $A_{2}$ and baryonium, which were both narrow features that appeared significant experimentally but which were evanescent [33]. So I have always advised lattice theorists not to rely on the experimental evidence for a pentaquark. A pentaquark state around $1540 \mathrm{MeV}$ has a "fall-apart" decay mode to $K N$. This decay does not involve any quark pair production so is expected to be unsuppressed - resulting in a very wide decay width (of the order of hundreds of $\mathrm{MeV}$ ).

Since the flimsy experimental evidence for the pentaquark is now weakening $[1,34,35]$, it is less compelling to study it exhaustively on the lattice. Nevertheless, it is instructive to discuss what lattice study could do to make more firm my conviction that "a narrow pentaquark is not possible in QCD". The essential issue is the narrowness of the claimed signal, since a broad resonance state 
would not be unexpected. In a lattice exploration of the pentaquark, an attractive phase shift in some $\mathrm{KN}$ channel is not sufficient to resolve the issue, the width needs to be evaluated. This needs, in principle,

- 2+1 flavours of sea quark, with light $u, d$.

- Operators to create multi-quark states and two-body states

- Vary spatial size to determine phase shift of two-body interaction.

Such a comprehensive study is not yet available. Instead most studies have used quenched QCD. The $K N$ decay channel is coupled in quenched studies to a pentaquark state, so this suggests that a quenched study can be a useful first step, although care must be taken of spurious contributions arising from states such as $K N \eta$ which will be relatively light and have unphysical behaviour. For realistic quark masses, there will be two body states on the lattice: their energy shifts then, following Lüscher, give the phase shift. By exploring this phase shift versus energy, the width of any resonance can then be extracted. In practice, for a very narrow resonance, the spectrum will look more like a one-body state (the resonance) and a collection of two-body states except for mixing near the avoided-level crossings. As the lattice volume is varied, the two body levels with non-zero momentum will move (since momentum is $2 \pi n / L$ ). Thus the volume dependence is a useful diagnostic. It is difficult, however, to determine accurately the many energy levels expected in a relatively large volume. An additional indicator is that the weight of the one-body and twobody contributions can be volume dependent: basically because the contribution from a two body state to a local-local correlator will be dominated by the lowest relative momentum which has a contribution which behaves as $1 / L^{3}$ compared to the contribution from a one-body state.

Using these criteria, lattice groups have explored the $\mathrm{S}$ - and P-wave $K N$ system. A summary of lattice results at LAT04 [36] showed most groups reproducing a pentaquark state. Many more results have been presented since and at this conference. Here I comment on some of the more comprehensive studies. The Kentucky group [37] use relatively light quarks and conclude no evidence for any pentaquark. One group which claimed lattice evidence for a pentaquark signal [38] has subsequently withdrawn their claim [39]. The analysis of quenched lattices at quark masses heavier than physical is still somewhat subjective and lattice evidence for a pentaquark is still being claimed by some groups, e.g. [40].

\section{Conclusions}

Hadronic physics involves unstable states.

Don't put your head in the sand: study these with lattice techniques.

We need to study interactions as well as spectra: solid gold - not just gold-plated.

\section{References}

[1] Particle Data Group Collaboration, S. Eidelman et. al., Review of particle physics (see http://pdg.lbl.gov), Phys. Lett. $\mathbf{B 5 9 2}$ (2004) 1.

[2] C. Michael, Shape of the $N^{*}(1236)$ resonance, Phys. Rev. 156 (1967) 1677-1684. 
[3] C. Michael, Particle decay in lattice gauge theory, Nucl. Phys. B327 (1989) 515.

[4] L. Maiani and M. Testa, Final state interactions from euclidean correlation functions, Phys. Lett. B245 (1990) 585-590.

[5] M. Luscher Commun. Math. Phys. 104 (1986) 177.

[6] M. Luscher Commun. Math. Phys. 105 (1986) 153.

[7] M. Luscher, Two particle states on a torus and their relation to the scattering matrix, Nucl. Phys. B354 (1991) 531-578.

[8] M. Luscher, Signatures of unstable particles in finite volume, Nucl. Phys. B364 (1991) 237-254.

[9] CP-PACS Collaboration, T. Yamazaki et. al., I = 2 pi pi scattering phase shift with two flavors of o(a) improved dynamical quarks, Phys. Rev. D70 (2004) 074513 [hep-lat / 0402025 ].

[10] NPLQCD Collaboration, S. R. Beane, P. F. Bedaque, K. Orginos and M. J. Savage, I = 2 pi pi scattering from fully-dynamical mixed-action lattice qcd, hep-lat/0506013.

[11] CP-PACS Collaboration, S. Aoki et. al., I = 2 pion scattering length from two-pion wave functions, Phys. Rev. D71 (2005) 094504 [hep-lat/ 0503025$].$

[12] UKQCD Collaboration, C. McNeile and C. Michael, Mixing of scalar glueballs and flavour-singlet scalar mesons, Phys. Rev. D63 (2001) 114503 [hep-lat/ 0010019 ].

[13] UKQCD Collaboration, P. Pennanen and C. Michael, String breaking in zero-temperature lattice $Q C D$, hep-lat/0001015.

[14] UKQCD Collaboration, C. McNeile and C. Michael, Hadronic decay of a vector meson from the lattice, Phys. Lett. B556 (2003) 177-184 [hep-lat/0212020].

[15] K. Rummukainen and S. A. Gottlieb, Resonance scattering phase shifts on a nonrest frame lattice, Nucl. Phys. B450 (1995) 397-436 [hep-lat/9503028].

[16] UKQCD Collaboration, C. McNeile, C. Michael and P. Pennanen, Hybrid meson decay from the lattice, Phys. Rev. D65 (2002) 094505 [hep-lat/ 0201006 ].

[17] C. Bernard et. al., Lattice calculation of 1-+ hybrid mesons with improved Kogut-Susskind fermions, Phys. Rev. D68 (2003) 074505 [hep-lat/ 0301024 ].

[18] M. Cook and R. Fiebig, Hybrid exotic meson decay width, PoS(LAT2005)062.

[19] C. Michael, Hadronic forces from the lattice, Nucl. Phys. Proc. Suppl. 26 (1992) 417-419.

[20] C. W. Bernard et. al., Zero temperature string breaking in lattice quantum chromodynamics, Phys. Rev. D64 (2001) 074509 [hep-lat/0103012].

[21] SESAM Collaboration, G. S. Bali, H. Neff, T. Duessel, T. Lippert and K. Schilling, Observation of string breaking in QCD, Phys. Rev. D71 (2005) 114513 [hep-lat/ 0505012 ].

[22] J. Sexton, A. Vaccarino and D. Weingarten, Numerical evidence for the observation of a scalar glueball, Phys. Rev. Lett. 75 (1995) 4563-4566 [hep-lat/9510022].

[23] J. Sexton, A. Vaccarino and D. Weingarten, Coupling constants for scalar glueball decay, Nucl. Phys. Proc. Suppl. 47 (1996) 128-135 [hep-lat/9602022].

[24] W.-J. Lee and D. Weingarten, Scalar quarkonium masses and mixing with the lightest scalar glueball, Phys. Rev. D61 (2000) 014015 [hep-lat/9910008].

[25] C. McNeile, C. Michael and J. Pickavance, in preparation, . 
[26] UKQCD Collaboration, A. M. Green, J. Koponen, C. McNeile, C. Michael and G. Thompson, Excited B mesons from the lattice, Phys. Rev. D69 (2004) 094505 [hep-lat / 0312007 ].

[27] UKQCD Collaboration, C. McNeile, C. Michael and G. Thompson, Hadronic decay of a scalar B meson from the lattice, Phys. Rev. D70 (2004) 054501 [hep-lat/ 0404010 ].

[28] UKQCD Collaboration, C. Michael and P. Pennanen, Two heavy-light mesons on a lattice, Phys. Rev. D60 (1999) 054012 [hep-lat/9901007].

[29] UKQCD Collaboration, A. Dougall, R. D. Kenway, C. M. Maynard and C. McNeile, The spectrum of $D_{s}$ mesons from lattice QCD, Phys. Lett. B569 (2003) 41-44 [hep-lat/ 0307001$]$.

[30] UKQCD Collaboration, A. M. Green, J. Koponen and C. Michael, P-wave radial distributions of a heavy-light meson on a lattice, AIP Conf. Proc. 756 (2005) 369-371 [hep-lat/ 0412002 ].

[31] J. Koponen, $B_{s}$ meson excited states from the lattice, PoS(LAT2005)205.

[32] C. W. Bernard et. al., The QCD spectrum with three quark flavors, Phys. Rev. D64 (2001) 054506 [hep-lat/0104002].

[33] T. Walcher, Experiments at the low-energy antiproton ring(LEAR), Ann. Rev. Nucl. Sci. 38 (1988) 67-95.

[34] News release 28 april 2005, JLAB.

[35] Belle Collaboration, K. Abe et. al., Search for the theta(1540)+ pentaquark using kaon secondary interactions at BELLE, hep-ex/0507014.

[36] S. Sasaki, Pentaquarks: Status and perspectives for lattice calculations, Nucl. Phys. Proc. Suppl. 140 (2005) 127-133 [hep-lat/0410016].

[37] N. Mathur et. al., A study of pentaquarks on the lattice with overlap fermions, Phys. Rev. D70 (2004) 074508 [hep-ph/0406196].

[38] F. Csikor, Z. Fodor, S. D. Katz and T. G. Kovacs, Pentaquark hadrons from lattice QCD, JHEP 11 (2003) 070 [hep-lat/0309090].

[39] F. Csikor, Z. Fodor, S. D. Katz, T. G. Kovacs and B. C. Toth, A comprehensive search for the theta+ pentaquark on the lattice, hep-lat/0503012.

[40] C. Alexandrou and A. Tsapalis, A lattice study of the pentaquark state, hep-lat/0503013. 